\title{
Anomalous metastability and fixation properties of evolutionary games on scale-free graphs
}

\author{
Michael Assaf ${ }^{1 \star}$ and Mauro Mobilia ${ }^{2 \star}$ \\ 1 Racah Institute of Physics, Hebrew University of Jerusalem \\ Jerusalem 91904, Israel \\ michael.assaf@mail.huji.ac.il \\ 2 Department of Applied Mathematics, School of Mathematics, University of Leeds \\ Leeds LS2 9JT, U.K. \\ M.Mobilia@leeds.ac.uk \\ http://www1.maths.leeds.ac.uk/ amtmmo/
}

\begin{abstract}
This contribution concerns the influence of scale-free graphs on the metastability and fixation properties of a set of evolutionary processes. In the framework of evolutionary game theory, where the fitness and selection are frequency-dependent and vary with the population composition, we analyze the dynamics of snowdrift games (characterized by a metastable coexistence state) on scale-free networks. Using an effective diffusion theory in the weak selection limit, we demonstrate how the scale-free structure affects the system's metastable state and leads to anomalous fixation. In particular, we analytically and numerically show that the probability and mean time of fixation are characterized by stretched exponential behaviors with exponents depending nontrivially on the network's degree distribution. Our approach is also shown to be applicable to models, like coordination games, not exhibiting metastability prior to fixation.
\end{abstract}

Keywords: Metastability, Fixation, Complex Networks, Evolutionary Games, Diffusion Theory

\section{Introduction \& background}

This contribution, based on Reference [1], is concerned with the influence of complex spatial structure on the metastability and fixation properties of a set of evolutionary processes characterized by frequency-dependent selection.

The dynamics of systems where successful traits spread at the expense of others is naturally modeled in the framework of evolutionary game theory (EGT) [2]. While the EGT classic setting was originally proposed to describe the evolution of infinitely large and spatially homogeneous populations, it is known that evolutionary dynamics is affected by demographic noise and by the population's spatial arrangement $[3,4]$. Evolutionary dynamics is often characterized by the central notion of fixation [5] that refers to the possibility that a "mutant type"

\footnotetext{
* The authors contributed equally to this work.
} 
takes over. In particular, one is interested in the probability that a given trait invades the entire population (fixation probability) and in the mean time for this event to occur (mean fixation time). In contrast to what happens in spatiallyhomogeneous (well-mixed) populations, the spatial arrangement of individuals can give rise to various very different scenarios [3]. In this context, evolutionary dynamics on networks [6] provides a general and unifying framework to describe the dynamics of both well-mixed and spatially-structured populations [7]. In spite of their importance, the fixation properties of evolutionary processes on networks have been mostly studied in idealized situations, e.g. for two-state systems under a constant and weak selective bias [7-10]. In these works, it has been shown that the update rules and the complex network structure effectively renormalize the population size and affect the system's fixation properties. Furthermore, some properties of evolutionary games have been studied on scale-free networks by numerical simulations, and on regular graphs with mean field and perturbative treatments, see e.g. [11]. The models of Refs. [7-10] are of great interest but do not provide a general description of evolutionary dynamics on graphs. In particular, these references consider constant fitness and selection pressure, and thus can not describe systems possessing a long-lived metastable coexistence state prior to fixation [12,13]. In our work [1], within the EGT framework, we have studied metastability, which may arise as a consequence of frequency-dependent selection, and fixation on a class of scale-free networks. To the best of our knowledge, such an analytical study has not been conducted before. For concreteness, this contribution mainly focuses on "snowdrift games" (SGs) [2] that are the paradigmatic evolutionary games exhibiting metastability and characterized by an exponential dependence on the population size on complete graphs (well-mixed populations), see e.g. [13]. Our findings are also directly relevant to various fields, e.g. to population genetics [14] and to the dynamics of epidemic outbreaks, for which a long-lived endemic state is often an intrinsic characteristic $[12,15]$.

Our central result, of broad relevance and importance, is the demonstration that evolutionary dynamics on scale-free networks can lead to anomalous fixation and metastability characterized by a stretched exponential dependence on the population size, in stark contrast with their non-spatial counterparts. We also show that such a dependence characterizes fixation in models like coordination games which do not possess a long-lived metastable state prior to fixation [2].

\section{The model}

We consider a network consisting of $N$ nodes, each of which is either occupied by an individual of type $\mathrm{C}$ (cooperator) or $\mathrm{D}$ (defector). The occupancy of the node $i$ is encoded by the random variable $\eta_{i}$, with $\eta_{i}=1$ if the node $i$ is occupied by a $C$ and $\eta_{i}=0$ otherwise. The state of the system is thus described by $\{\eta\}=\left\{\eta_{i}\right\}^{N}$

and the density of cooperators present in the system is $\rho \equiv \sum_{i=1}^{N} \eta_{i} / N$. The adjacency matrix $\mathbf{A}=\left[A_{i j}\right]$ of this simple and undirected network is symmetric with elements $A_{i j}=1$ if the nodes $i j$ are connected and 0 otherwise [6]. The 
network is also characterized by its degree distribution $n_{k}=N_{k} / N$, where $N_{k}$ is the number of nodes of degree $k$. For a generic two-strategy cooperation dilemma, the payoff of $C$ against another $C$ is denoted $a$ and that of $D$ playing against $D$ is $d$. When $C$ and $\mathrm{D}$ play against each other, the former gets payoff $b$ and the latter gets $c$. For snowdrift games, in which we are chiefly interested, one has $c>a$ and $b>d$ and the (mean field) dynamics is characterized by two unstable absorbing states $\rho=0$ and $\rho=1$ separated by a (meta-)stable interior fixed point $\rho_{*}=(d-b) /(a-b-c+d)$. In a spatial setting, the interactions are among nearest-neighbor individuals and the species payoffs are defined locally: $C$ and $D$ players at node $i$ interacting with a neighbor at site $j$ respectively receive payoffs $\Pi_{i j}^{C}=a \eta_{j}+b\left(1-\eta_{j}\right)$ and $\Pi_{i j}^{D}=c \eta_{j}+d\left(1-\eta_{j}\right)$. In the spirit of the Moran model (with weak selection pressure) $[4,5]$, each species local reproductive potential, or fitness, is given by the difference of $\Pi_{i j}^{C / D}$ relative to the population mean payoff $\bar{\Pi}_{i j}(t)$. Here, we make the mean-field-like choice $\bar{\Pi}_{i j}(t)=\rho(t) \Pi_{i j}^{C}+(1-\rho(t)) \Pi_{i j}^{D}$ to include what arguably is the simplest mechanism ensuring the formation of metastability. It is customary to introduce a selection strength $s>0$ in the definition of the fitness to unravel the interplay between random fluctuations and selection $[4,5]$. By introducing a baseline contribution set to unity, the fitnesses of C/D at node $i$ interacting with a neighbor at site $j$ are $f_{i j}^{C}=1+s\left[\Pi_{i j}^{C}-\bar{\Pi}_{i j}\right]$ and $f_{i j}^{D}=1+s\left[\Pi_{i j}^{D}-\bar{\Pi}_{i j}\right]$, respectively. The system evolves according to the "link dynamics" (LD) [8,9]: a link is randomly selected at each time step and, if it connects a CD pair, one of the neighbors is randomly selected for reproduction with a rate proportional to its fitness while the other is replaced by the offspring. While various types of update rules are possible, we have used the LD for the sake of simplicity and to highlight the combined effects of the topology and frequency-dependent selection. However, we have checked that our conclusion is robust and holds for various other update rules leading to metastability [1].

\section{Methodology}

The system's evolutionary dynamics is analyzed in terms of $\left\{\rho_{k}\right\}$, where $\rho_{k}=$ $\sum_{i}^{\prime} \eta_{i} / N_{k}$ is the average number of cooperators on all nodes of degree $k$ (the prime means that the sum is restricted to nodes of degree $k$ ). Hence, $\rho_{k}$ is the subgraph density of C's on nodes of degree $k$. The analysis also relies on the $m^{t h}$-moment of the degree distribution, denoted $\mu_{m} \equiv \sum_{k} k^{m} n_{k}$, and the degree-weighted density of cooperators $\omega \equiv \sum_{k}\left(k / \mu_{1}\right) n_{k} \rho_{k}$. The analytical treatment thus proceeds in five steps, (a)-(e):

(a) Birth-death process formulation: In our model, the subgraph densities $\left\{\rho_{k}\right\}$ evolve according to a (continuous-time) birth-death process defined by the transition rates $T_{k}^{+}=\sum_{i}^{\prime} \sum_{j} A_{i j} \Psi_{i j} / \bar{N}$ and $T_{k}^{-}=\sum_{i}^{\prime} \sum_{j} A_{i j} \Psi_{j i} / \bar{N}$, where $\Psi_{i j}=\left(1-\eta_{i}\right) \eta_{j} f_{j i}^{C}, \Psi_{j i}=\left(1-\eta_{j}\right) \eta_{i} f_{j i}^{D}$ and $\bar{N} \equiv N \mu_{1}$. At each time-increment $\delta t=N^{-1}$, the subgraph density $\rho_{k}$ thus respectively changes by $\pm \delta \rho_{k}=$ $\pm 1 / N_{k}$ according to the transition rates $T_{k}^{ \pm}$. In our analytical calculations, we have focused on degree-heterogeneous networks (of finite mean degree $\mu_{1}$ ) 
with degree-uncorrelated nodes, see e.g. [17], yielding $A_{i j}=k_{i} k_{j} / \bar{N}$, while numerical simulations were performed using the "redirection algorithm" [18].

(b) Multivariate Fokker-Planck equation:

In the limit of weak selection intensity $(s \ll 1$ ) [13], one can use the diffusion theory to investigate the properties of the above birth-death process [5]. This yields a multivariate backward Fokker-Planck equation (FPE) whose generator reads [16]

$$
\begin{aligned}
& \mathcal{G}\left(\left\{\rho_{k}\right\}\right)=\sum_{k}\left[\frac{\left(T_{k}^{+}-T_{k}^{-}\right)}{n_{k}} \frac{\partial}{\partial \rho_{k}}+\frac{\left(T_{k}^{+}+T_{k}^{-}\right)}{2 N n_{k}^{2}} \frac{\partial^{2}}{\partial \rho_{k}^{2}}\right], \quad \text { with } \\
& T_{k}^{+}=\left(n_{k} / \mu_{1}\right)[1+s(b-d)(1-\rho)] k\left(1-\rho_{k}\right) \omega \quad \text { and } \\
& T_{k}^{-}=\left(n_{k} / \mu_{1}\right)[1-s(a-c) \rho] k \rho_{k}(1-\omega)
\end{aligned}
$$

(c) Time scale separation:

While the multivariate FPE generated by (1) is generally not tractable, when the selection intensity is weak $(s \ll 1)$, a time scale separation allows to greatly simplify the mathematical analysis $[1,9,10]$. Indeed, when $t \ll s^{-1}$ the selection pressure is negligible and the quantity $\rho$ is conserved [8], before relaxing to its metastable value $\rho_{*}$ on a time scale $t \sim s^{-1} \gg 1$. Furthermore, as shown in [1] and illustrated in Fig. 1, after a time of order $\mathcal{O}(1)$, one finds that on average all subgraph densities $\bar{\rho}_{k}$ and the mean degree-weighted density $\bar{\omega}$ approach the average density $\bar{\rho}$ and all these quantities approach $\rho_{*}$ on a time scale $t \sim s^{-1}$.

(d) Effective single-coordinate Fokker-Planck equation:

Since fixation occurs on much longer time scales than $s^{-1}$, when $\bar{\rho}_{k} \approx \bar{\omega} \approx \bar{\rho}$ (weak selection) and the fluctuations about the metastable state are small, one can use the approximation $\rho_{k} \approx \rho \approx \omega$ in the generator (1) of the backward FPE $[1,9]$. Hence, with the change of variables $\rho_{k} \rightarrow \omega$ and $\rho_{k} \approx$ $\rho \approx \omega$, Eq. (1) yields the effective single-coordinate FPE generator $[1,9]$

$$
\mathcal{G}_{\text {eff }}(\omega)=\frac{\omega(1-\omega)}{N_{\text {eff }}}\left[-\sigma\left(\omega-\rho_{*}\right) \frac{\partial}{\partial \omega}+\frac{1}{2} \frac{\partial^{2}}{\partial \omega^{2}}\right],
$$

where $\sigma \equiv 2(b-d) N_{\text {eff }} s_{\text {eff }} / \rho_{*}[1]$. The complex spatial structure renormalizes the population size and selection intensity, yielding the effective quantities $N_{\text {eff }}=N\left(\mu_{1}\right)^{3} / \mu_{3}$ and $s_{\text {eff }} \equiv s \mu_{2} /\left(\mu_{1}\right)^{2}$, whose expressions depend on the first three moments of the degree distribution. In the effective FPE generator (2), the drift term is thus proportional to $\sigma \propto s N \mu_{1} \mu_{2} / \mu_{3}$. For scale-free networks with degree distribution $n_{k} \sim k^{-\nu}$ and finite average degree (i.e. $\nu>2$ ), the maximum degree is $k_{\max } \sim N^{1 /(\nu-1)}$ [19]. From the moments $\mu_{m}$, one thus obtains the nontrivial scaling of $\sigma$ with the population size [1]:

$$
\sigma \propto s N \frac{\mu_{1} \mu_{2}}{\mu_{3}} \sim \sigma_{\mathrm{re}}= \begin{cases}s N, & \nu>4 \\ s N / \ln N, & \nu=4 \\ s N^{(2 \nu-5) /(\nu-1)}, & 3<\nu<4 \\ s \sqrt{N} \ln N, & \nu=3 \\ s N^{(\nu-2) /(\nu-1)}, & 2<\nu<3 .\end{cases}
$$




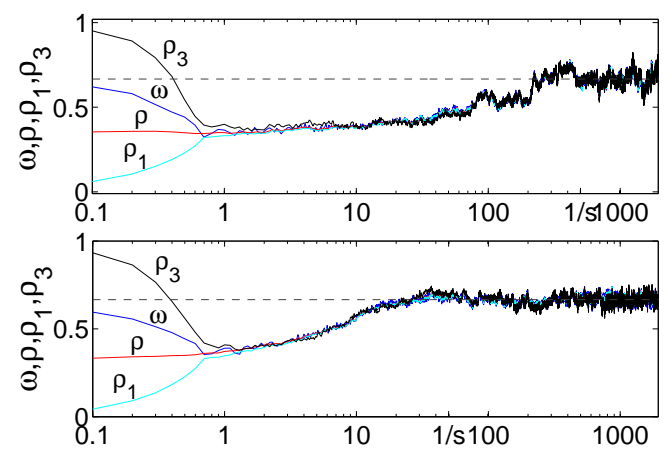

Fig. 1. (Adapted from [1]). Time scale separation in typical Monte Carlo trajectories of the densities $\rho, \omega, \rho_{1}$, and $\rho_{3}$ on a scale-free network with $\nu=3$ for a SG with $a=d=1, b=9, c=5$ and $N=10^{4}$. The selection intensity is $s=0.002$ (top) and $s=0.02$ (bottom), and initially $\rho_{k>\mu_{1}}(0)=1, \rho_{k \leq \mu_{1}}(0)=0$. One notices two time scales: after a time of order $\mathcal{O}(1)$, the trajectories of $\rho, \omega, \rho_{1}$, and $\rho_{3}$ almost coincide and evolve together toward their common metastable value $\rho_{*}=2 / 3$ (dashed) that is reached after a time of order $t \sim s^{-1}$ (indicated on the horizontal axis). Fixation occurs on a much longer time scale, see text and Ref. [1].

(e) Calculation of the fixation properties:

In the realm of the effective diffusion approximation (2), the fixation probability $\phi^{C}(\omega)$ that a system with initial degree-weighted density $\omega$ is taken over by cooperators is obtained analytically by solving the backward FPE $\mathcal{G}_{\text {eff }}(\omega) \phi^{C}(\omega)=0$ with boundary conditions $\phi^{C}(0)=1-\phi^{C}(1)=0$ [16]. Similarly, the unconditional mean (fixation) time $\tau$ to reach an absorbing boundary is given by the solution of $\mathcal{G}_{\text {eff }}(\omega) \tau(\omega)=-1$, with $\tau(0)=\tau(1)=0$.

\section{Results}

The analytical results of this research have been corroborated by stochastic simulations and concern the thorough analysis of the influence of the scalefree network structure on the metastability and fixation properties of a class of evolutionary processes (snowdrift and coordination games).

\subsection{Implications and validity of the effective theory}

We have shown that the scale-free structure is responsible for a nontrivial renormalization of the population size and selection intensity in an important class of evolutionary ("snowdrift") games. In the limit of weak selection, the properties of these models are accurately described by the effective diffusion theory (2)-(3). To appreciate the implications of the nontrivial scaling (3), it is instructive to 
consider the scale-free graphs with $2<\nu<3$ that are characterized by the divergence of $\mu_{2}$ and $\mu_{3}$ (when $N \rightarrow \infty$ ). For this class of complex networks that include nodes of high degree, we have found $N_{\text {eff }}=N^{(2 \nu-5) /(\nu-1)} \ll N$ and $s_{\text {eff }}=s N^{(3-\nu) /(\nu-1)} \gg s$. In this case, the fluctuations intensity $\left(\propto N_{\text {eff }}^{-1 / 2}\right)$ and the drift strength $\left(\propto s_{\text {eff }}\right)$ are both enhanced by the complex topology. Yet, the product of these effective quantities yields $N_{\text {eff }} s_{\text {eff }} \sim s N^{(\nu-2) /(\nu-1)} \ll N s$, which implies that the scale-free topology can drastically reduce the mean fixation time [1], see below.

We have checked that the predictions of the effective theory are accurate when $s_{\text {eff }} \ll N_{\text {eff }}^{-1}$ (e.g. when $s^{2} \ll N^{-1 /(\nu-1)}$ for $2<\nu<3$ ). Hence, when $2<\nu<4$, the effective diffusion approximation is applicable for a broader range of values of $s$ than in a non-spatial setting (where the requirement is $s^{2} \ll N^{-1}[13]$ ).

\subsection{Fixation probability in snowdrift games}

Evolutionary dynamics is characterized by the fixation probability $\phi^{C}(\omega)$ that a system with initial degree-weighted density $\omega$ is taken over by cooperators.
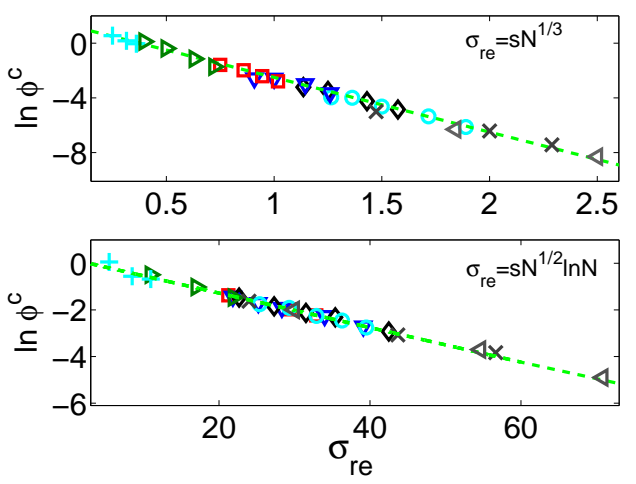

Fig. 2. (Adapted from [1]). Scaling of the fixation probability: $\ln \phi^{C}$ versus $\sigma_{\text {re }}$ for a SG with $a=d=1, b=1.05, c=1.075$ [scenario (i)] on scale-free graphs with $\nu=2.5$ (top) and $\nu=3$ (bottom). Numerical results have been obtained for $s=0.025(+)$, $0.05(\triangleright), 0.075(\square), 0.1(\nabla), 0.125(\diamond), 0.15(\circ), 0.2(\times), 0.25(\triangleleft)$, see text and Ref. [1]. Here, $N=400-4000$ and initially $\rho_{k}=\rho=\omega=100 / N$.

As described in Sec. 3(e), we have analytically computed the fixation probability in the realm of the effective diffusion theory (2)-(3) and found

$$
\phi^{C}(\omega)=\frac{\operatorname{erfi}\left[\rho_{*} \sqrt{\sigma}\right]-\operatorname{erfi}\left[\left(\rho_{*}-\omega\right) \sqrt{\sigma}\right]}{\operatorname{erfi}\left[\rho_{*} \sqrt{\sigma}\right]+\operatorname{erfi}\left[\left(1-\rho_{*}\right) \sqrt{\sigma}\right]},
$$

where $\operatorname{erfi}(z) \equiv \frac{2}{\sqrt{\pi}} \int_{0}^{z} e^{u^{2}} d u$. In Ref. [1], we have carefully discussed the biologically relevant case where $\omega \ll 1$ (low initial density of cooperators), the 
population size is large with $\sigma \gg 1$, and the selection pressure is weak $[4,5]$. In this situation, metastability (long-lived coexistence) is guaranteed and the fixation probability is characterized by a stretched-exponential dependence on the population size according to two main cases (when $\omega \ll 1$ ):

(i) $\ln \phi^{C} \simeq-\left(1-2 \rho_{*}\right) \sigma$, when $\rho_{*}<1 / 2$

(ii) $\ln \left(1-\phi^{C}\right) \simeq-\omega\left(2 \rho_{*}-\omega\right) \sigma$, if $\rho_{*}>1 / 2$ and $\omega<2 \rho_{*}-1$,

where the asymptotic dependence of $\sigma \propto s N \mu_{1} \mu_{2} / \mu_{3}$ is given by (3). The result (4) is corroborated by the stochastic simulations of Fig. 2 where results for various values of $s$ and $N$ have been rescaled to test the scaling relation (3). The linear data collapse and clear stretched-exponential dependence observed in Fig. 2 (see [1] for further results) confirm the predictions of (3) and (4). This demonstrates that the fixation probability of a few mutants may be drastically enhanced on a scale-free graph (with respect to a non-spatial setting).

\subsection{Mean fixation time in snowdrift games}

Metastability and evolutionary dynamics are also characterized by the mean fixation time (MFT) $\tau(\omega)$, i.e. the (unconditional) mean time necessary to reach an absorbing boundary.
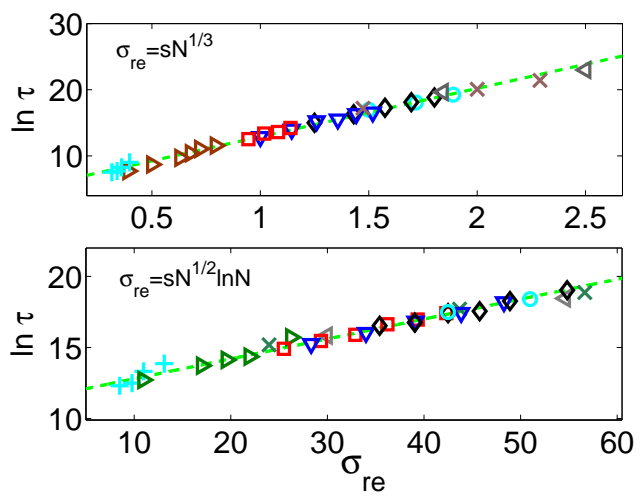

Fig. 3. (Adapted from [1]). Scaling of the mean fixation time: $\ln \tau$ versus $\sigma_{\text {re }}$ for $\nu=2.5$ (top) and $\nu=3$ (bottom). Symbols and parameters are as in Fig. 2. Numerical results collapse along the dashed line, in agreement with (5). Initially $\rho_{k}=\rho=0.5$, see text.

As discussed in Sec. 3(e), we have solved the effective backward FPE for the unconditional mean fixation time $\tau$ and, to leading order in $\sigma \gg 1$, have found that $\tau$ exhibits a stretched-exponential dependence on $N$ [1]:

$$
\tau(\omega) \sim \begin{cases}\left(1-\phi^{C}(\omega)\right) e^{\sigma \rho_{*}^{2}}, & \text { when } \omega>\rho_{*} \\ \phi^{C}(\omega) e^{\sigma\left(1-\rho_{*}\right)^{2}}, & \text { otherwise. }\end{cases}
$$


With (4) and (5), one finds the leading-order contribution to the MFT: e.g., when $\sigma \gg 1$ and $\rho_{*}<1 / 2, \tau$ is found to grow with $N$ as the stretched exponential $\ln \tau \simeq \sigma \rho_{*}^{2}$. This prediction is confirmed by Fig. 3 (see Ref. [1] for further simulation results) and implies that on scale-free networks with degree distribution $n_{k} \sim k^{-\nu}$ and $2<\nu<4$, fixation occurs much more rapidly than on complete graphs. This phenomenon is called "hyperfixation" in population genetics [14].

\subsection{Fixation probability in coordination games}

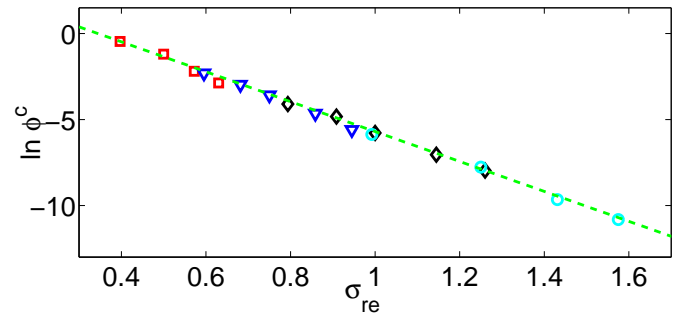

Fig. 4. Logarithm of the fixation probability $\ln \phi^{C}$ versus $\sigma_{\mathrm{re}}(3)$ for a CG with $a=$ $1.6, b=c=1$ and $d=1.2\left(\rho_{*}=0.75\right)$ on a scale-free graph with $\nu=2.5$. Here, $s=0.05(\square), 0.075(\triangle), 0.1(\diamond)$ and $0.125(\circ), N=1000-4000$ and $\sigma_{k}(0)=0.5$. Numerics are consistent with the theoretical prediction $\ln \phi^{C} \sim-s N \mu_{1} \mu_{2} / \mu_{3} \simeq-s N^{1 / 3}$, see text.

We have also studied the class of "coordination games" (CGs) for which $a>c$ and $d>b$. In CGs, the interior fixed point $\rho_{*}$ is unstable, whereas the absorbing states $\rho=0$ and $\rho=1$ are stable (bistability) [2]. While there is no metastability in CGs, the fixation probability in coordination games evolving with the link dynamics on scale-free graphs has been found to have the same stretched-exponential dependence on $N$ as in snowdrift games (see also [13]), as shown in Fig. 4.

\section{Conclusion}

We have studied metastability and fixation of evolutionary processes on scalefree networks. For the sake of concreteness, we have focused on the paradigmatic case of "snowdrift games". The probability and mean fixation time have been computed from an effective Fokker-Planck equation derived by exploiting a time scale separation in the weak selection limit. These quantities exhibit a stretched-exponential dependence on the population size, in stark contrast with their non-spatial counterparts. We have checked with various update rules that the stretched-exponential behavior is a generic feature of metastability on scalefree graphs that also characterizes the fixation probability of coordination games. 
Important consequences of the stretched-exponential behavior are a drastic reduction of the mean fixation time, and the possible enhancement of the fixation probability of a few mutants with respect to a non-spatial setting.

\section{References}

1. M. Assaf and M. Mobilia, e-print arXiv:1202.3231v2 (http://arxiv.org/abs/1202 . 3231). To appear in Physical Review Letters (2012).

2. J. Maynard Smith, Evolution and the Theory of Games (Cambridge University Press, Cambridge, 1982); J. Hofbauer and K. Sigmund, Evolutionary Games and Population Dynamics (Cambridge University Press, Cambridge, 1998); M. A. Nowak, Evolutionary Dynamics (Belknap Press, 2006); G. Szabó and G. Fáth, Phys. Rep. 446, 97 (2007).

3. M. A. Nowak and R. M. May, Nature 359, 826 (1992); C. Hauert and M. Doebeli, Nature 428, 643 (2004); M. Nowak, Science 314, 1560 (2006).

4. M. A. Nowak et al., Nature 428, 646 (2004).

5. J. F. Crow and M. Kimura, An Introduction to Population Genetics Theory (Blackburn Press, New Jersey, 2009); W. J. Ewens, Mathematical Population Genetics (Springer, New York, 2004).

6. A. L. Barabási and R. Albert, Science 286, 509 (1999); R. Albert and A. L. Barabási, Rev. Mod. Phys. 74, 47 (2002); M. E. J. Newman,Networks:An Introduction (Oxford University Press, New York, 2010).

7. E. Lieberman et al., Nature 433, 312 (2005); H. Ohtsuki et al., Nature 441, 502 (2006).

8. C. Castellano, D. Vilone, and A. Vespignani, EPL 63, 153 (2003); K. Suchecki, V. M. Eguiluz, M. San Miguel, EPL 69, 228 (2005).

9. V. Sood and S. Redner, Phys. Rev. Lett. 94, 178701 (2005); T. Antal et al., Phys. Rev. Lett. 96, 188104 (2006); V. Sood et al., Phys. Rev. E 77, 041121 (2008);

10. G. J. Baxter, R. A. Blythe, and A. J. McKane, Phys. Rev. Lett. 101, 258701 (2008); R. A. Blythe, J. Phys. A: Math. Theor. 43, 385003 (2010).

11. F. C. Santos and J. M. Pacheco, Phys. Rev. Lett. 95, 098104 (2005); H. Ohtsuki and M. A. Nowak, J. Theor. Biol. 243, 86 (2006), C. E. Tarnita et al., J. Theor. Biol. 259, 570 (2009).

12. M. Assaf and B. Meerson, Phys. Rev. Lett. 97, 200602 (2006); Phys. Rev. E 81, $155912(2010)$.

13. M. Mobilia and M. Assaf, EPL 91, 10002 (2010); M. Assaf and M. Mobilia, J. Stat. Mech., P09009 (2010).

14. P. A. Wigham et al., Th. Pop. Biol. 74, 283 (2008).

15. R. Pastor-Satorras and A. Vespignani, Phys. Rev. Lett. 86, 3200 (2001); I. Nåsell, J. Theor. Biol. 211, 11 (2001); R. M. May and A. L. Lloyd, Phys. Rev. E 64, 066112 (2001); M. E. J. Newman, Phys. Rev. E 66, 016128 (2002); R. Durrett, Proc. Nat. Acad. Sci. USA 107, 4491 (2010).

16. C. W. Gardiner, Handbook of Stochastic Methods, (Springer, New York, 2002).

17. M. Molloy and B. Reed, Random. Struct. Algoritms 6, 161 (1995).

18. S. N. Dorogovtsev, J. F. F. Mendes, and A. N. Samukhin, Phys. Rev. Lett. 85, 4633 (2000); P. L. Krapivsky and S. Redner, Phys. Rev. E 63, 066123 (2001).

19. P. L. Krapivsky and S. Redner, J. Phys. A 35, 9517 (2002). 